\title{
Evaluation of the SLICS use in the treatment of subaxial cervical spine injuries
}

\author{
Avaliação do SLICS no tratamento das lesões da coluna cervical sub-axial \\ Halisson Y. F. da Cruz', Andrei F. Joaquim', Helder Tedeschi', Alpesh A. Patel ${ }^{2}$
}

\begin{abstract}
The SLICS (Sub-axial Cervical Spine Injury Classification System) was proposed to help in the decision-making of sub-axial cervical spine trauma (SCST), even though the literature assessing its safety and efficacy is scarce. Method: We compared a cohort series of patients surgically treated based on surgeon's preference with patients treated based on the SLICS. Results: From 2009-10, 12 patients were included. The SLICS score ranged from 2 to 9 points (mean of 5.5). Two patients had the SLICS < 4 points. From 2011-13, 28 patients were included. The SLICS score ranged from 4 to 9 points (mean of 6 ). There was no neurological deterioration in any group. Conclusion: After using the SLICS there was a decrease in the number of patients with less severe injuries that were treated surgically. This suggests that the SLICS can be helpful in differentiating mild from severe injuries, potentially improving the results of treatment.
\end{abstract}

Keywords: sub-axial cervical spine, SLICS, spinal cord injury, spine trauma, classification, treatment.

\section{RESUMO}

O SLICS (Sub-axial Cervical Spine Injury Classification System) foi proposto para auxílio na tomada de decisão no tratamento do traumatismo da coluna cervical sub-axial. Contudo, existem poucos trabalhos que avaliem sua segurança e eficácia. Método: Realizamos estudo comparativo de série histórica de pacientes operados baseados na indicação pessoal do cirurgião com pacientes tratados baseados na aplicação do SLICS. Resultados: Entre 2009-10, 12 pacientes foram incluídos. O SLICS escore variou de 2 a 9 pontos (média de 5,5) com dois pacientes com escore menor que 4. Entre 2011-13, 28 pacientes foram incluídos. O escore de SLICS variou de 4 a 9 pontos, com média de 6. Conclusão: Observamos que após o uso do SLICS houve uma diminuição do número de pacientes operados com lesões mais estáveis. Isso sugere que o SLICS pode ser útil para auxiliar a diferenciação de lesões leves das graves, eventualmente melhorando os resultados do tratamento.

Palavras-chave: traumatismo da coluna vertebral, SLICS, lesão medular, trauma de coluna, classificação, tratamento.

Cervical spine trauma can potentially result in serious neurological injury such as tetraplegia or severe disability. Sub-axial cervical spine trauma (SCST), involving the spine levels of $\mathrm{C} 3$ to $\mathrm{C} 7$, accounts for the majority of cervical spine injuries, comprising about $65 \%$ of fractures and $75 \%$ of all dislocations that affects the spine ${ }^{1}$.

In an attempt to improve clinical results and compare treatment modalities, numerous classification systems that describe these injuries, try to predict stability, and also help with the choice of the treatment to be performed ${ }^{2,3,4}$. However, no one of them is universally accepted. One potential reason for the lack of a universal classification is that the treatment of SCST is based on a number of variables that include fracture patterns, suspected mechanism of injury, spinal alignment, neurologic injury, and expected long-term stability, which difficults reliability and reproducibility.

Considering this, the Spine Trauma Study group proposed the Sub-axial Cervical Spine Injury Classification System (SLICS). This system is based on the evaluation of three major injury characteristics as follows: (1) injury morphology, determined by the pattern of spinal column disruption on available imaging studies, (2) integrity of the discoligamentous soft tissue complex (DLC) represented by both anterior and posterior ligamentous structures as well as the intervertebral disc, and (3) patient's neurologic status. The system proposes a severity score grading, from the least to the most severe injury pattern ${ }^{1}$. The final score can help in the choice of conservative versus surgical treatment. The presence of morphological

${ }^{1}$ Universidade Estadual de Campinas, Departamento de Neurologia, Campinas SP, Brazil;

${ }^{2}$ Northwestern University, Department of Orthopedic, Chicago IL, USA.

Correspondence: Halisson Y F da Cruz; Rua Olinda Alves, 819 / casa 17 - Rita Vieira; 79052-440 Campo Grande MS, Brasil; E-mail: halissonyoshinari@hotmail.com Conflict of interest: There is no conflict of interest to declare.

Received 14 November 2014; Received in final form 06 january 2015; Accepted 26 January 2015. 
abnormalities is scored as: 0- no abnormality, 1- compression, 2- burst fractures, 3- distraction, and 4- translation or rotation. The components of the DLC include the intervertebral disc, anterior and posterior longitudinal ligaments, interspinous ligaments, facet capsules, and ligamentum flavum. The integrity of these soft tissue constraints is directly proportional to spinal stability and is scored as 0- for intact, 1 - for indeterminate and 2- with disruption. Neurologic injury is the third component of the SLIC system and is inherently an important indicator of the severity of spinal column injury and may be the single most influential predictor of treatment. Patients neurologically intact receive 0- point, 1- for cervical root injury, 2- for complete neurological deficit, and the presence of an incomplete neurologic injury receives the highest point score of 3 . In the presence of ongoing root or cord compression the authors proposed an additional 1 point. Additionally, confounding factors can influence the treatment decision including medical comorbidities, presence of ankylosing spondylitis, diffuse idiopathic hyperostosis, osteoporosis, previous surgery, and degenerative disease ${ }^{1}$. The SLICS score is presented in Table 1.

Surgical versus non-surgical treatment is suggested by a threshold value of the SLICS score. If the total score is $<4$ (1 to 3), non-operative treatment is recommended. If the total is $\geq 5$, operative treatment is recommended. This treatment may consist of realignment, neurological decompression (when indicated), and stabilization. Cases with a total score of 4 may be treated either operatively or non-operatively based upon surgeon and patient preferences.

Based on the potential benefits of the score, the purpose of this study is to evaluate the impact of SLICS in patients treated surgically for SCST.

Table 1. The Subaxial Cervical Spine Injury Classification System.

\begin{tabular}{lc}
\hline Morphology & Points \\
\hline No abnormality & 0 \\
Compression & 1 \\
Burst & 2 \\
Distraction (e.g., facet perch, hyperextension) & 3 \\
Rotation/translation (e.g., facet dislocation, unstable & 4 \\
teardrop or advanced staged flexion compression injury) & \\
Discoligamentous complex (DLC) & \\
Intact & 0 \\
Indeterminate (e.g., isolated interspinous widening, & 1 \\
MRI signal change only) & \\
Disrupted (e.g., widening of the disc space, facet & 2 \\
perch, or dislocation) & \\
Neurological status & \\
Intact & 0 \\
Root injury & 1 \\
Complete cord injury & \\
Incomplete cord injury & \\
Continuous cord compression in setting of neuro \\
deficit ("neuromodifier")
\end{tabular}

MRI: Magnetic resonance imaging.

\section{METHOD}

The spine trauma database at the University of Campinas, Campinas-SP, Brazil was used. The institution is a tertiary trauma center. There were two group of patients:

Group 1: From 2009 to 10, these patients were treated according to the treating surgeon's preference, based on personal decisions for conservative versus surgical treatment but not guided by the SLICS score. The SLICS was applied retrospectively on this group;

Group 2: From 2011-13, the SLICS was used to guide the treatment (patients with four or more points were referred to surgical treatment).

\section{Inclusion criteria}

Patient's age (> 17), presence of sub-axial cervical injury treated surgically (main level of trauma from C3 to C7), and complete radiological and clinical data for retrospective application of the SLICS. The medical records and radiological data were considered adequate for retrospective application of the SLIC when the three injury characteristics of the SLIC could be scored properly, with a CT scan or a CT and MRI.

\section{Exclusion criteria}

Incomplete radiographic or clinical data, pathological fractures (infection, cancer), isolated upper cervical trauma (occiput to $\mathrm{C} 2$ ), isolated transverse process or spinous process fractures, chronic or age determinate fractures, isolate MRI findings, and severe systemic trauma with death prior to surgical treatment.

\section{Demographic data included}

Age, gender, injury characteristics and treatment details were recorded, including trauma ethiology, fracture level (in segmental trauma we considered the upper vertebra as the level of injury), neurological status, surgical approach and complications. Follow-up included clinical assessment of the neurological status, at least one post-operatory CT scan with reconstruction to check instrumentation and serial plain radiographs at 1, 3, 6 months and them anually.

The SLICS from 2009 to 10 was scored retrospectively based on clinical and radiological data reviewed by two authors (HYFC and AFJ). From 2011-13, the SLICS was applied by AFJ.

Neurological status was scored according to the American Spinal Injury Association (ASIA) Impairment Scale (AIS) in: complete neurological deficit (AIS A), incomplete (AIS B, C or D), or intact (AIS E) $)^{5}$.

Confounding factors (e.g., ankylosing spondylitis, diffuse idiopathic hyperostosis, osteoporosis, previous surgery, and degenerative disease) were noted if present.

Outcomes of treatment during follow-up, the approach used, as well as, complications directed related to the surgical procedure (neurological deficit and surgical complications) were recorded. Institutional ethical committee approval was obtained prior to initiation of the study (129/2011). 


\section{RESULTS}

A total of 12 patients were surgically treated and had total radiological and clinical data for inclusion on this study from 2009-10. From 2011-13, twenty eight patients were treated surgically based on the SLIC score and were included.

\section{Surgical group 2009-2010 - (12 patients)}

From a total of 21 operated patients from 2009-10, only 12 cases surgically treated were included because considering our inclusion criteria. Nine (75\%) were male and $3(25 \%)$ were female. Table 2 summarizes the results of this group.

The follow up ranged from 1.8 to 65.5 months (mean of 24.5). Patient's age ranged from 17 to 60 years (mean 29.5).

Regarding the level of injury, one patient (8.3\%) had injury at C4, $4(33.3 \%)$ at C5, $5(41.6 \%)$ at C6, $2(16.6 \%)$ at $C 7$ and no one at $\mathrm{C} 3$. In two patients the trauma was caused by motor vehicle rollover and in one case by a motorcycle accident. Another two patients had falls from heights, four patients were hit by cars and three had dived into shallow water.

Preoperatively, 5 patients (41.6\%) were AIS E, 1 (8.3\%) AIS D, 1 (8.3\%) AIS C, 1 (8.3\%) AIS B and 4 (33.3\%) AIS A. No patients had neurological worsening. At the final follow up, two patients (28.5\% of the patients with neurological deficits) improved the AIS status (AIS B to C and B to D).

The SLICS score in this group ranged from 2 to 9 points (mean of 5.5, median of 5.75 and $\mathrm{SD} \pm 2.05$ ). Two patients $(16.6 \%)$ with a SLICS of less than 4 points were operated, one with SLICS of 2 points ( 2 burst +0 for DLC +0 for neurological status) and other with a SLICS of 3 points ( 0 for morphology +0 for DLC +3 for incomplete neurologic injury - AIS D). Four patients (33.3\%) had 5 points, two (16.6\%) 6 points, one (8.3\%) 7 points, two (16.6\%) 8 points, and one $(8,3 \%)$ had 9 points.

Six patients (50\%) underwent an anterior approach and the other six (50\%) underwent a posterior approach, with the objective of spinal realignment, stabilization and decompression. Combined approaches (anterior and posterior) were not used from 2009-10.

Complications included postoperative wound infection after instrumented posterior cervical fusion in 1 patient (neurologically intact) and one tracheoesophageal fistula requiring direct surgical repair of the esophagus. There were no deaths.

\section{Surgical group 2011-2013 - (28 patients)}

Data from the 28 cases of cervical spine trauma treated surgically based on the SLICS score from 2011-2013 is presented in Table 3. Follow-up was obtained in all 28 patients, ranging from 0.1 to 24 months (mean of 6.1).

Patients' age ranged from 20 to 82 years (mean 41.5). Twenty-five (89.3\%) were male and $3(10.7 \%)$ were female.

Regarding the level of injury, 8 patients $(28.6 \%)$ had the trauma at C3, $5(17.9 \%)$ at C4, $7(25 \%)$ at C5, $8(28.6 \%)$ at $\mathrm{C} 6$ and no one at $\mathrm{C} 7$. The main cause of trauma was car
Table 2. Summary of the 12 patients surgically treated from 2009-2010.

\begin{tabular}{lcccccc}
\hline N & Sex & Age & Level & AIS & SLICS & Complications \\
\hline 1 & M & 31 & C6 & A & 7 & Infection \\
2 & M & 38 & C5 & E & 5 & No \\
3 & M & 53 & C4 & E & 5 & No \\
4 & F & 28 & C6 & E & 5 & No \\
5 & M & 20 & C5 & B to C & 8 & No \\
6 & M & 17 & C5 & B to D & 8 & Tracheoesophageal fistula \\
7 & F & 43 & C5 & D & 3 & No \\
8 & M & 23 & C6 & E & 2 & No \\
9 & M & 60 & C6 & C & 9 & No \\
10 & M & 43 & C7 & E & 6 & No \\
11 & F & 21 & C7 & A & 5 & No \\
12 & M & 22 & C6 & A & 6 & \\
\hline
\end{tabular}

Table 3. Summary of the 28 patients surgically treated from 2011-2013.

\begin{tabular}{|c|c|c|c|c|c|c|}
\hline $\mathrm{N}$ & Sex & Age & Level & ASIA & SLICS & Complication \\
\hline 1 & $\mathrm{M}$ & 26 & C3 & A & 7 & No \\
\hline 2 & M & 22 & C3 & A & 8 & No \\
\hline 3 & $\mathrm{~F}$ & 48 & C6 & $E$ & 5 & No \\
\hline 4 & M & 23 & C4 & E & 5 & No \\
\hline 5 & M & 65 & C6 & C & 8 & No \\
\hline 6 & M & 40 & C5 & $E$ & 6 & No \\
\hline 7 & M & 45 & C6 & $E$ & 5 & No \\
\hline 8 & $\mathrm{~F}$ & 71 & $\mathrm{C} 6$ & B to $\mathrm{C}$ & 8 & No \\
\hline 9 & $\mathrm{~F}$ & 48 & C6 & E & 7 & Infection \\
\hline 12 & M & 65 & C6 & C & 6 & Deep venous thrombosis \\
\hline 13 & M & 46 & C5 & E & 5 & No \\
\hline 14 & M & 40 & C3 & A & 4 & No \\
\hline 15 & M & 82 & C5 & B to $C$ & 9 & No \\
\hline 16 & M & 29 & C5 & $D$ to $E$ & 5 & No \\
\hline 17 & M & 28 & C6 & E & 5 & No \\
\hline 18 & M & 23 & $\mathrm{C} 4$ & A & 8 & Dural injury \\
\hline 19 & M & 49 & $\mathrm{C} 4$ & E & 6 & No \\
\hline 20 & M & 45 & $\mathrm{C} 4$ & E & 7 & No \\
\hline 21 & M & 20 & C5 & D & 7 & No \\
\hline 22 & M & 43 & C3 & C to D & 4 & No \\
\hline 23 & M & 56 & C5 & E & 7 & No \\
\hline 24 & M & 54 & $\mathrm{C} 3$ & $C$ to $D$ & 6 & No \\
\hline 25 & M & 32 & $\mathrm{C} 4$ & E & 6 & No \\
\hline 26 & M & 40 & $\mathrm{C} 3$ & $D$ & 5 & No \\
\hline 27 & M & 23 & C6 & $E$ & 6 & No \\
\hline 28 & M & 51 & $\mathrm{C} 3$ & C to D & 5 & No \\
\hline
\end{tabular}

accidents in twelve patients (42.9\%), nine patients (32.1\%) fell from heights, in three cases (10.7\%) the cause was motorcycle accidents, two $(7.15 \%)$ dove into shallow water and two (7.15\%) had direct traumas.

Preoperatively, 12 patients (42.9\%) were AIS E, 4 (14.3\%) AIS D, 5 (17.9\%) AIS C, 2 (7.15\%) AIS B and 5 (17.9\%) AIS A. No patients had neurological worsening.

At the final follow up, the AIS score was: 13 (46.64\%) AIS E, 6 (21.4\%) AIS D, 4 (14.3\%) AIS C, and 5 (17.9\%) AIS A. 
A total of six out of eleven (54.5\%) patients with incomplete neurological deficit (AIS B-C-D) improved their ASIA status during the follow-up (Table 3).

The SLICS score in this group ranged from 4 to 9 points (mean of 6 , median of 6 and SD \pm 1.4 ). The two patients with a SLICS of 4 points had a central cord syndrome without fractures or dislocations.

Twelve patients (42.9\%) underwent an anterior approach, thirteen $(46.4 \%)$ a posterior approach and three $(10.7 \%)$ a combined approach. No preoperative traction was used in any case of this series.

Complications directed related with surgery in this group included one wound infection (posterior approach) requiring surgical debridement. One patient had a deep venous thrombosis prior to surgery and received an inferior vena cava filter and another patient the diagnosis of an intraoperative dural tear treated with fibrin glue and muscle graft.

\section{DISCUSSION}

Despite the technological advances in surgical techniques for spinal instrumentation, classification of SCST remains largely descriptive, lacking standardization and usually correlates poorly with clinical outcomes ${ }^{1}$.

Historically, one of the first comprehensive classification systems for spinal injuries was credited to Holdsworth ${ }^{2}$. His system was the first to emphasize the importance of the posterior ligamentous complex (PLC) in long term stability (noteworthy, the evaluation of the ligamentous complex is one of the three main categories evaluated by the SLICS).

In 1982, Allen et al. ${ }^{3}$ subsequently proposed their mechanistic classification scheme for subaxial injuries, which was also based upon the findings of plain radiographs. A total of six main categories were defined by Allen: compressive flexion, vertical compression, distractive flexion, compressive extension, distractive extension, and lateral flexion. A potential and important limitation of the Allen's classification is considering only plain radiographs to interpret the mechanism of injuries, which can result in low reliability and poor clinical outcome relationship ${ }^{6}$.

The AO Spine group also extrapolated their thoracolumbar system for cervical injuries, classifying them into three main groups: group A, with compression and burst fractures, group $\mathrm{B}$ with distractive injuries and group $\mathrm{C}$, with rotational injuries. This system is widely adopted and can help surgeons in describing injuries, although it is criticized by not considering the role of the neurological status in the decision-making process?

The sub-axial injury classification system (SLICS) was developed by Vaccaro et al. to define a classification system for SCST that conveys information about injury patterns and severity as well as treatment considerations and prognosis, such as neurological status and the role of the ligamentous complex in long-term stability ${ }^{1,8,9}$.

The SLICS had already demonstrated validity in previous studies: in a retrospective clinical study of patients with SCST treated, 14 patients were treated non-surgically (C), whereas 24 were treated surgically (S). In the C group, the SLIC score ranged from 0 to 5 points (mean 1.07; median 1). Just one patient had an SLIC score greater than 2 (7.1\% of the patients). In the S group, the SLIC score ranged from 1 to 10 points (mean 5.6; median 6). Just 2 patients had an SLICS score smaller than 4 (both with 1 point each, $8.3 \%$ of the total group). All the other 22 (accounting for 91.6\%) patients had an SLICS of 4 or more points. The SLIC score matched the treatment chosen (non surgical or surgical) with more than $90 \%$ of agreement between them ${ }^{10}$.

A prospective application of the SLICS in a consecutive series of 37 patients with SCST to define injuries and guide surgical decision is also reported. Patients with four or more points were surgically treated, whereas patients with less than 4 points were conservatively managed. Twenty-three patients were included in the non-surgical group: 14 (61\%) of them with some follow-up at the original institution. Follow-up ranged from three to five months (mean of 4.42; median 4). The SLICS score ranged from 0 to 6 points (mean and median of 1 ). One patient with a SLICS of 6 points refused surgery ${ }^{11}$.

In the surgical group: twenty-five patients were operated, follow-up after hospital discharge was obtained in 23 (92\%) patients (range from one to 24 months, mean of 5.82 months). The SLICS score in the surgical group ranged from 4 to 9 points (mean and median of 7). No patients had neurological worsening. Eight out of 13 patients with incomplete deficits had some improvement in the ASIA score. In this study the SLICS system was identified as being safe and effective at preventing neurological deterioration and, in most patients, led to clinically relevant improvements in neurological function ${ }^{11}$.

Although our study has some limitations, such as relative small sample and single center involved, we could observe a trend that, after the use of the SLICS, there were no patients operated with low injury score (group 2011-2013) compared with 2 of 12 patients (16.67\%) treated with mild injuries (group 2009-10). This can suggest that SLICS may help surgeons with the standardization of care as well as with the choice of more unstable patterns for surgical treatment.

The SLICS is a comprehensive and useful tool to guide SCST treatment by spine surgeons. Larger prospective multicenter studies including conservative and surgically treated patients are necessary to access benefits in patient's outcome with its use. 


\section{References}

1. Vaccaro AR, Hulbert RJ, Patel AA, Fisher C, Dvorak M, Lehman Jr RA et al. The subaxial cervical spine injury classification system: a novel approach to recognize the importance of morphology, neurology, and integrity of the disco-ligamentous complex. Spine (Phila Pa 1976) 2007;32(21):2365-74. http://dx.doi.org/10.1097/BRS.0b013e3181557b92

2. Holdsworth F. Fractures, dislocations, and fracture-dislocations of the spine. J Bone Joint Surg Am. 1970;52:1534-51.

3. Allen Jr BL, Ferguson RL, Lehmann TR. A mechanistic classification of closed, indirect fractures and dislocations of the lower cervical spine. Spine (Phila Pa 1976) 1982;7:1-27.

4. Harris JH, Edeiken-Monroe B, Kopansiky DR. A practical classification of acute cervical spine injuries. Orthop Clin North Am.1986;17(1):15-30.

5. Marino RJ, Barros T, Biering-Sorensen F, Burns SP, Donovan WH, Graves DE et al. International standards for neurological classification of spinal cord injury.J Spinal Cord Med. 2003;26(1):50-6.

6. Whang PG, Patel AA, Vaccaro AR. The development and evaluation of the subaxial injury classification scoring system for cervical spine trauma. Clin Orthop Relat Res. 2011;469:723-31. http://dx.doi.org/10.1007/s11999-010-1576-1

7. Magerl F, Aebi M, Gertzbein SD, Harms J, Nazarian S. A comprehensive classification of thoracic and lumbar injuries. Eur Spine J. 1994;3(4):184-201. http://dx.doi.org/10.1007/BF02221591

8. Joaquim AF, Patel AA, Vaccaro AR. Cervical injuries scored according to the subaxial injury classification system: an analysis of the literature. J Craniovertebr Junction Spine. 2014;5(2):65-70. http://dx.doi.org/10.4103/0974-8237.139200

9. Joaquim AF, Patel AA. Subaxial cervical spine trauma: evaluation and surgical decision-making. Global Spine J. 2014;4(1):63-70. http://dx.doi.org/10.1055/s-0033-1356764

10. Joaquim AF, Lawrence B, Daubs M, Brodke D, Patel AA. Evaluation of the subaxial injury classification system. J Craniovertebr Junction Spine. 2011;2(2):67-72. http://dx.doi.org/10.4103/0974-8237.100057

11. Joaquim AF, Ghizoni E, Tedeschi H, Cruz HY, Patel AA. Clinical results of patients with subaxial cervical spine trauma treated according to the SLIC score. J Spinal Cord Med. 2013;37(4):4204. http://dx.doi.org/10.1179/2045772313Y.0000000143 